\title{
Quantum Monte Carlo study of the ground state of the two-dimensional Fermi fluid
}

\author{
N. D. Drummond and R. J. Needs \\ TCM Group, Cavendish Laboratory, University of Cambridge, J. J. Thomson Avenue, Cambridge CB3 OHE, United Kingdom
}

(Received 24 June 2008; published 13 February 2009)

\begin{abstract}
We have used the variational and diffusion quantum Monte Carlo methods to calculate the energy, paircorrelation function, static structure factor, and momentum density of the ground state of the two-dimensional (2D) homogeneous electron gas. We have used highly accurate Slater-Jastrow-backflow trial wave functions and twist averaging to reduce finite-size effects where applicable. We compare our results with others in the literature and construct a local-density-approximation exchange-correlation functional for 2D systems.
\end{abstract}

DOI: 10.1103/PhysRevB.79.085414

PACS number(s): 71.10.Ca, 71.10.Pm, 71.10.Ay

\section{INTRODUCTION}

The homogeneous electron gas (HEG) plays a key role in modern condensed-matter theory. It consists of a set of electrons moving in a uniform, inert neutralizing background and is the simplest fully interacting quantum many-body model of condensed matter. The three-dimensional HEG models the behavior of the conduction electrons in metals and semiconductors, while the two-dimensional (2D) HEG models the behavior of electrons confined to surfaces and thin layers. Quantum Monte Carlo (QMC) methods ${ }^{1,2}$ have long played an important role in establishing the ground-state properties of the HEG. Most of the effort has gone into calculating the ground-state energy of different phases as a function of density in order to establish the zero-temperature phase diagram. ${ }^{3-5}$ In this article, we report QMC calculations of some other properties of the 2D HEG of interest to condensed-matter physicists: the pair-correlation function (PCF), static structure factor (SSF), and momentum density (MD). We also report energy data for high-density HEGs. We have confined our attention to the fluid phase, which is the ground state at the densities typically encountered in experiments.

The PCF, especially the contact PCF $g(0)$, is a key ingredient in generalized-gradient-approximation exchangecorrelation functionals for density-functional theory (DFT) calculations. The PCF has been studied several times using QMC, ${ }^{3,4,6,7}$ but the value of $g(0)$ at low densities has proved controversial because electrons approach one another infrequently, and the QMC results disagree with values calculated using ladder theory. ${ }^{8}$ Our QMC data, obtained using a different trial wave function from the earlier calculations, should help to clarify the situation. The SSF is related to the PCF by a Fourier transform. SSF data at small $k$ are needed to establish the long-range behavior of the PCF.

The MD of the HEG is of considerable importance in Fermi-liquid theory. To our knowledge, the only QMC MD data to have been published for the 2D HEG are those of Tanatar and Ceperley, ${ }^{3}$ which used a relatively simple form of trial wave function. (A fit to QMC data generated by Conti ${ }^{9}$ is reported in Ref. 10, but no details about the calculations are given.) The low-density MD of Tanatar and Ceperley $^{3}$ shows a very strange feature: the MD is lower at zero momentum than it is at the top of the Fermi edge. It is clearly important to provide different QMC MD data in order to establish whether this is a genuine property of the HEG.

Finally, we report ground-state energy data for paramagnetic Fermi fluids, which we use to parametrize a localdensity-approximation exchange-correlation functional to use in DFT studies of 2D systems.

The rest of this article is arranged as follows. In Sec. II we describe the computational techniques used. In Sec. III we present the data we have generated. Finally, we draw our conclusions in Sec. IV. Densities are given in terms of the radius $r_{s}$ of the circle that contains one electron on average. We use Hartree atomic units $\left(\hbar=|e|=m_{e}=4 \pi \epsilon_{0}=1\right)$ throughout this article. All our QMC calculations were performed using the CASINO code. ${ }^{11}$

\section{QMC CALCULATIONS}

\section{A. Trial wave functions}

In the variational quantum Monte Carlo (VMC) method, expectation values are calculated with respect to an approximate trial wave function, the integrals being performed by a Monte Carlo technique. In diffusion quantum Monte Carlo ${ }^{1,2}$ (DMC) the imaginary-time Schrödinger equation is used to evolve an ensemble of electronic configurations toward the ground state. The fermionic symmetry is maintained by the fixed-node approximation ${ }^{12}$ in which the nodal surface of the wave function is constrained to equal that of a trial wave function. The VMC algorithm generates electron configurations distributed according to the square of the trial wave function, while the DMC algorithm generates configurations distributed as the product of the trial wave function and its ground-state component.

Our trial wave functions consisted of Slater determinants of plane-wave orbitals multiplied by a Jastrow correlation factor. The Jastrow factor contained polynomial and planewave expansions in electron-electron separation. ${ }^{13}$ The orbitals in the Slater wave function were evaluated at quasiparticle coordinates related to the actual electron positions by backflow functions consisting of polynomial expansions in electron-electron separation. ${ }^{14}$ The wave functions were optimized by variance minimization ${ }^{15,16}$ and linear-leastsquares energy minimization. ${ }^{17}$

We simulated HEGs in finite square cells subject to periodic boundary conditions. The many-body Bloch theorem ${ }^{18}$ states that the wave function $\Psi$ satisfies 
TABLE I. Energy, variance, and percentage of correlation energy retrieved using different methods for a 58-electron paramagnetic Fermi fluid of density parameter $r_{s}=5$ a.u. Twist averaging has not been used. "HF," "SJ-VMC," "SJB-VMC," "SJ-DMC," and "SJB-DMC" stand for Hartree-Fock theory, VMC with a SlaterJastrow wave function, VMC with a Slater-Jastrow-backflow wave function, DMC with a Slater-Jastrow wave function, and DMC with a Slater-Jastrow-backflow wave function, respectively. The DMC energy data have been extrapolated to zero time step. The data marked with an asterisk were produced by Kwon et al. (Ref. 19), while the data marked with a dagger were generated by Attaccalite et al. (Ref. 21) at a time step of 0.1 a.u. (i.e., their data were not extrapolated to zero time step). The fraction of the correlation energy retrieved is computed on the assumption that our SlaterJastrow-backflow DMC calculation retrieves $100 \%$ of the correlation energy.

\begin{tabular}{lccc}
\hline \hline Method & $\begin{array}{c}\text { Energy } \\
\text { (a.u./elec.) }\end{array}$ & $\begin{array}{c}\text { Var. } \\
\text { (a.u.) }\end{array}$ & Frac. corr. en \\
\hline HF & -0.100222006 & & $0 \%$ \\
SJ-VMC & $-0.1482110(8)$ & 0.0196 & $96.910(4) \%$ \\
SJ-VMC & $-0.14680(5)$ & & $94.1(1) \%$ \\
SJB-VMC & $-0.1493851(6)$ & 0.00774 & $99.282(4) \%$ \\
SJB-VMC & -0.148 80(5) & & $98.1(1) \%$ \\
SJ-DMC & $-0.149177(8)$ & & $98.86(2) \%$ \\
SJ-DMC & $-0.14900(5)$ & & $98.5(1) \%$ \\
SJ-DMC & $-0.149134(9)$ & & $98.77(2) \%$ \\
SJB-DMC & $-0.149741(2)$ & & $100 \%$ \\
SJB-DMC* & $-0.14955(5)$ & & $99.6(1) \%$ \\
SJB-DMC ${ }^{\dagger}$ & $-0.149518(9)$ & & $99.55(2) \%$ \\
\hline \hline
\end{tabular}

$$
\Psi\left(\mathbf{r}_{1}, \ldots, \mathbf{r}_{i}+\mathbf{R}_{s}, \ldots, \mathbf{r}_{N}\right)=\exp \left(i \mathbf{k}_{s} \cdot \mathbf{R}_{s}\right) \Psi\left(\mathbf{r}_{1}, \ldots, \mathbf{r}_{N}\right),
$$

where $\mathbf{R}_{s}$ is a simulation-cell lattice vector and $\mathbf{k}_{s}$ is the simulation-cell Bloch vector. In some of our calculations, and in previous QMC studies of the 2D HEG, ${ }^{3,4,19}$ it has been assumed that $\mathbf{k}_{s}=\mathbf{0}$. However, in our calculations of the energy, PCF, and SSF we performed twist averaging (TA) in which expectation values are averaged over $\mathbf{k}_{s}$ in the first Brillouin zone of the simulation cell. ${ }^{20}$ This procedure greatly reduces single-particle finite-size errors caused by shell-filling effects.

The high quality of our trial wave functions is demonstrated in Table I, which shows QMC energies achieved using different levels of wave function for a 58-electron paramagnetic Fermi fluid of density parameter $r_{s}=5$ a.u. Backflow functions change the nodal surface of the trial wave function and can therefore improve the fixed-node DMC energy. In practice we find that backflow lowers the DMC energy substantially. Our VMC energies are significantly lower than those of Kwon et al., ${ }^{19}$ as is our SlaterJastrow-backflow DMC energy. On the other hand, the Slater-Jastrow-backflow DMC energy of Attaccalite et al. ${ }^{21}$ is higher than that of Kwon et al. ${ }^{19}$ Our Slater-Jastrow DMC energy is slightly lower than that of Attaccalite et al., ${ }^{21}$ which in turn is lower than that of Kwon et al. ${ }^{19}$ Since the nodal surface is the same in the three calculations, these DMC energies really ought to agree. However, the trial wave function used by Kwon et al. ${ }^{19}$ is very much poorer than ours, as can be seen by comparing the VMC energies. Timestep and population-control biases in their DMC energies must be much greater, which may explain the discrepancy. The results of Attaccalite et al. ${ }^{21}$ have not been extrapolated to zero time step, hindering comparison. The VMC and DMC results of Rapisarda and Senatore ${ }^{4}$ are in very close agreement with those of Kwon et al. ${ }^{19}$

We have optimized a three-electron term in the Jastrow factor (together with the two-electron Jastrow terms and backflow functions) for a paramagnetic 58-electron HEG at $r_{s}=5$ a.u. The three-electron term lowered the nontwistaveraged VMC energy from -0.149385 1(6) to $-0.1495111(5)$ a.u. per electron. The DMC energies at a time step of 0.1 a.u. without and with the three-body Jastrow factor are $-0.149742(2)$ and $-0.149740(2)$ a.u. per electron, respectively. As expected, the inclusion of the threebody term makes an insignificant difference to the DMC energy because the DMC energy depends only on the nodal surface of the trial wave function, which is not directly affected by the Jastrow factor. We have therefore not used three-electron terms in our production calculations.

\section{B. Evaluating expectation values}

\section{Evaluating the $M D$}

Let $\Psi(\mathbf{R})$ be the trial many-electron wave function, where $\mathbf{R}=\left(\mathbf{r}_{1}, \ldots, \mathbf{r}_{N}\right)$. Suppose that the first $N_{\uparrow}$ electrons are spin up and the remaining electrons are spin down. The MD of spin-up electrons can be evaluated as

$$
\rho(\mathbf{k})=\left\langle\frac{1}{(2 \pi)^{3}} \int \frac{\Psi\left(\mathbf{r}, \mathbf{r}_{2}, \ldots, \mathbf{r}_{N}\right)}{\Psi(\mathbf{R})} \exp \left[i \mathbf{k} \cdot\left(\mathbf{r}_{1}-\mathbf{r}\right)\right] d \mathbf{r}\right\rangle,
$$

where the angled brackets denote an average over the set of electron configurations generated in the VMC and DMC algorithms (which are distributed as $|\Psi|^{2}$ and $\left|\Psi \Phi_{0}\right|$, respectively, where $\Phi_{0}$ is the ground-state component of $\left.\Psi\right)$. We have restricted our attention to paramagnetic and fully ferromagnetic HEGs, so the total MD is equal to the spin-up MD. The integral in the expectation value of Eq. (2) is estimated by Monte Carlo sampling at each configuration $\mathbf{R}$ generated by the QMC algorithms, and the results are averaged. The use of a finite number of points in the evaluation of the integral at each $\mathbf{R}$ does not bias the QMC estimate of $\rho(\mathbf{k})$.

Suppose our finite simulation cell has area $A$ and the simulation-cell Bloch vector is $\mathbf{k}_{s}$. We may write

$$
\frac{\Psi\left(\mathbf{r}, \mathbf{r}_{2}, \ldots, \mathbf{r}_{N}\right)}{\Psi(\mathbf{R})}=\frac{1}{A} \sum_{\mathbf{G}} c_{\mathbf{G}}(\mathbf{R}) \exp \left[i\left(\mathbf{G}+\mathbf{k}_{s}\right) \cdot \mathbf{r}\right],
$$

where the $\{\mathbf{G}\}$ are the simulation-cell reciprocal lattice points. Hence it is clear that $\rho(\mathbf{k})$ is only nonzero if $\mathbf{k}=\mathbf{G}$ $+\mathbf{k}_{s}$ for some $\mathbf{G}$. The MD is only defined for a discrete set of momenta at any given $\mathbf{k}_{s}$. One cannot twist average as such; instead, altering $\mathbf{k}_{s}$ leads to the MD being defined at a dif- 
ferent set of momenta. We simply report MDs obtained using $\mathbf{k}_{s}=\mathbf{0}$ (i.e., no twist was applied).

\section{Evaluating the SSF}

The SSF may be evaluated as

$$
S(\mathbf{k})=\frac{1}{N}[\langle\hat{n}(\mathbf{k}) \hat{n}(-\mathbf{k})\rangle-\langle\hat{n}(\mathbf{k})\rangle\langle\hat{n}(-\mathbf{k})\rangle],
$$

where

$$
\hat{n}(\mathbf{k})=\sum_{i} \exp \left(-i \mathbf{k} \cdot \mathbf{r}_{i}\right)
$$

is the Fourier transform of the density operator. $S(\mathbf{k})$ is only nonzero at simulation-cell $\mathbf{G}$ vectors, even if the simulationcell Bloch vector is nonzero. We can twist average when we calculate $S(\mathbf{G})$.

\section{Evaluating the PCF}

The spherically averaged PCF is

$$
g(r)=\frac{\Omega}{4 \pi r^{2} N^{2}}\left\langle\sum_{i \neq j} \delta\left(\left|\mathbf{r}_{i}-\mathbf{r}_{j}\right|-r\right)\right\rangle,
$$

which can be evaluated by binning the electron-electron distances in the configurations generated by the QMC algorithms. Twist averaging introduces no complications.

\section{Extrapolated estimation}

If $\hat{A}$ is an operator that does not commute with the Hamiltonian then the errors in the VMC and DMC estimates $A_{\mathrm{VMC}}$ and $A_{\mathrm{DMC}}$ of the expectation value of $\hat{A}$ are linear in the error in the trial wave function; however, the error in the extrapolated estimate $2 A_{\mathrm{DMC}}-A_{\mathrm{VMC}}$ is quadratic in the error in the trial wave function. ${ }^{2}$ We have used extrapolated estimation in most of our calculations of expectation values. Examples of extrapolation are shown in Figs. 5 and 8, and the upper panel of Fig. 4. In each case the VMC, DMC, and extrapolated estimates are in good agreement, implying that the error resulting from the use of a DMC mixed estimate is small. Gori-Giorgi et al. ${ }^{7}$ used reptation ${ }^{22} \mathrm{QMC}$ to accumulate the PCF and SSF in which pure expectation values are obtained with respect to the fixed-node ground-state wave function so that extrapolation is unnecessary.

\section{Time-step and population-control biases}

Finite-time-step errors in the twist-averaged DMC energy were removed by linear extrapolation to zero time step. An example is shown in Fig. 1; it can be seen that the time-step bias is in fact very small in any case. We checked that the other expectation values were converged with respect to the time step: see Figs. 4 and 5. We used a target population of 1600 configurations in all our DMC calculations, making population-control bias negligible.

\section{Finite-size bias}

Expectation values obtained in a finite $N$-electron cell subject to periodic boundary conditions (PBC) differ from

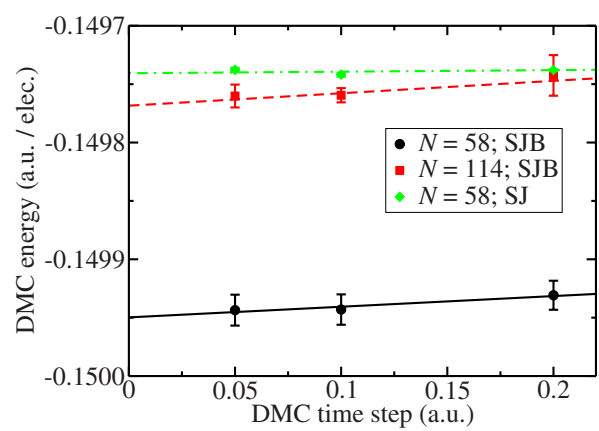

FIG. 1. (Color online) Twist-averaged DMC energy against time step for paramagnetic Fermi fluids of density parameter $r_{s}=5$ a.u. at different system sizes $N$. "SJ" and "SJB" refer to Slater-Jastrow and Slater-Jastrow-backflow wave functions, respectively.

the corresponding infinite-system values because of "singleparticle" shell-filling effects, as well as the neglect of longranged correlations and the compression of the exchangecorrelation hole into the simulation cell. Single-particle finite-size effects can be removed from the energy, the SSF, and the PCF by twist averaging, as explained in Sec. II A. We have recently demonstrated that the finite-size error in the energy per particle in a 2D HEG falls off as $N^{-5 / 4}$, enabling us accurately to extrapolate QMC energies to infinitesystem size. ${ }^{23}$ For the PCF, SSF, and MD we simply verified that the QMC data had converged with respect to system size (see Figs. 5 and 8, and the lower panel of Fig. 4).

\section{RESULTS}

\section{A. Energies}

DMC energies of paramagnetic Fermi fluids at different densities and system sizes are shown in Table II. Our results for the energies of different phases of the 2D HEG at low density are reported elsewhere. ${ }^{5}$ At $r_{s}=5$ and 10 a.u., Rapisarda and Senatore ${ }^{4}$ obtained infinite-system energies of

TABLE II. Twist-averaged DMC energy, extrapolated to zero time step, for $N$-electron paramagnetic Fermi fluids of density parameter $r_{s}$. Where $N=\infty$, the DMC energy has been extrapolated to infinite-system size.

\begin{tabular}{lcl}
\hline \hline $\begin{array}{l}r_{s} \\
\text { (a.u.) }\end{array}$ & $N$ & $\begin{array}{c}\text { DMC energy } \\
\text { (a.u./elec.) }\end{array}$ \\
\hline 1 & 50 & $-0.2125(2)$ \\
1 & 74 & $-0.2122(2)$ \\
1 & 114 & $-0.2116(3)$ \\
1 & $\infty$ & $-0.2104(6)$ \\
5 & 58 & $-0.14995(2)$ \\
5 & 114 & $-0.14977(1)$ \\
5 & $\infty$ & $-0.14963(3)$ \\
10 & 58 & $-0.085504(5)$ \\
10 & 74 & $-0.08552(1)$ \\
10 & 114 & $-0.085445(3)$ \\
10 & $\infty$ & $-0.085399(6)$ \\
\hline \hline
\end{tabular}


TABLE III. Fitting parameters in Eq. (7) for the correlation energy of a paramagnetic HEG. Equation (7) was fitted to the infinite-system DMC energies given in Table II and the low-density energy data given in Ref. 5. It was found that fixing $H_{0}=0$ did not affect the quality of the fit.

\begin{tabular}{ll}
\hline \hline Parameter & \multicolumn{1}{c}{ Value } \\
\hline$A_{0}$ & -0.1925 \\
$B_{0}$ & 0.086313631 \\
$C_{0}$ & 0.06979568 \\
$D_{0}$ & 0.0 \\
$E_{0}$ & 1.0531003 \\
$F_{0}$ & 0.04069122 \\
$G_{0}$ & 0.3605953 \\
$H_{0}$ & 0.0 \\
\hline \hline
\end{tabular}

$-0.1490(1)$ and $-0.08512(2)$ a.u. per particle using DMC with a Slater-Jastrow wave function. Kwon et al. ${ }^{19}$ obtained DMC energies of $-0.2098(3), \quad-0.1495(1)$, and $-0.08536(2)$ a.u. per electron at $r_{s}=1,5$, and 10, respectively, using a Slater-Jastrow-backflow wave function. Our DMC energies are somewhat lower than these data, as expected from the results shown in Table I.

Let the correlation energy per electron $E_{c}$ be the difference between the ground-state energy per electron and the Hartree-Fock (HF) energy. We fit the form proposed by Attaccalite et $a .^{21}$ to our correlation energies for paramagnetic HEGs as follows:

$$
\begin{aligned}
E_{c}= & A_{0}+\left(B_{0} r_{s}+C_{0} r_{s}^{2}+D_{0} r_{s}^{3}\right) \\
& \times \log \left(1+\frac{1}{E_{0} r_{s}+F_{0} r_{s}^{3 / 2}+G_{0} r_{s}^{2}+H_{0} r_{s}^{3}}\right),
\end{aligned}
$$

where $A_{0}=-0.1925, B_{0}=\sqrt{2}(10-3 \pi) /(3 \pi)$, and $D_{0}=-A_{0} H_{0}$. We fit to the infinite-system DMC energies shown in Table II and also to the DMC energies of low-density paramagnetic HEGs reported in Ref. 5 (at $r_{s}=20,25,30,35$, and 40 a.u.). Our fitting parameters are shown in Table III, and the correlation energies of paramagnetic Fermi fluids obtained by different authors relative to that of Attaccalite et al. ${ }^{21}$ are shown in Fig. 2. Our correlation energies are lower than those of the other authors because of our use of flexible backflow functions. Equation (7) can be used as a local-densityapproximation exchange-correlation functional in DFT calculations for 2D systems.

Unlike Attaccalite et al., ${ }^{21}$ we fit Eq. (7) to paramagnetic data only; we do not attempt to calculate the spinpolarization dependence of the energy of the HEG.

\section{B. MDs}

The MDs of paramagnetic HEGs are shown in Fig. 3, and a more detailed graph of the MD at $r_{s}=5$ a.u. is shown in Fig. 4. The upper panel of Fig. 4 demonstrates that the extrapolated estimate is accurate and that the DMC results are converged with respect to the time step. It is clear from the lower panel of Fig. 4 that, although backflow makes a sig-

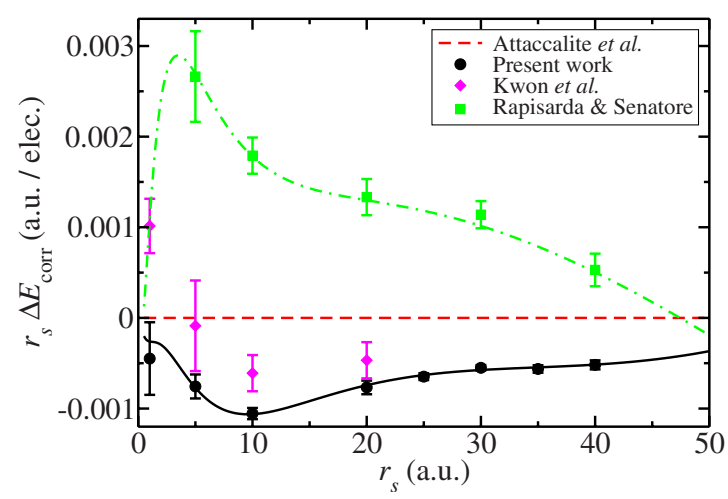

FIG. 2. (Color online) Correlation energy of a paramagnetic Fermi fluid relative to the results of Attaccalite et al. (Ref. 21). The results obtained by Kwon et al. (Ref. 19) and Rapisarda and Senatore (Ref. 4) are shown for comparison. The results of Tanatar and Ceperley (Ref. 3) are not shown because they are systematically too low in energy (Ref. 19).

nificant improvement to the QMC energy estimates, it has very little effect on the MD. The inclusion of backflow results in a small transfer of weight to wave vectors above the Fermi edge, as expected, because a greater fraction of correlation energy is retrieved. It can also be seen that the MDs obtained at different system sizes are in agreement. We have therefore plotted data obtained at different system sizes together in Fig. 3. To our knowledge, the only previous QMC studies of the MD of the 2D HEG are those of Tanatar and Ceperley $^{3}$ and Conti. ${ }^{9}$ At $r_{s}=10$ a.u., Tanatar and Ceperley ${ }^{3}$ found that the MD at small wave vectors is lower than the value near the Fermi edge. Our data do not show this unusual feature. Tanatar and Ceperley $^{3}$ used a relatively inflexible Slater-Jastrow wave function, which may be the reason for the discrepancy. Giuliani and Vignale ${ }^{10}$ quoted a formula for the MD, which was obtained by fitting to QMC data generated by Conti. ${ }^{9}$ We have fitted our MDs to a simplified version of the form suggested in Ref. 10,

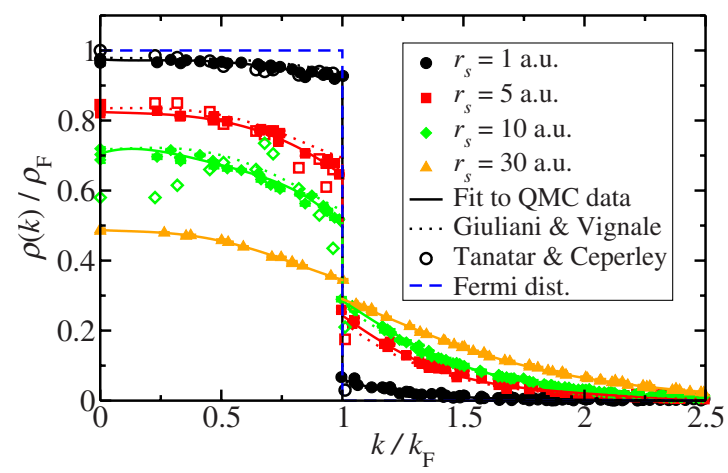

FIG. 3. (Color online) Extrapolated MD $\rho(k)$ for paramagnetic Fermi fluids. $k_{F}=\sqrt{2} / r_{s}$ is the Fermi wave vector of the paramagnetic fluid and $\rho_{F}=r_{s}^{2} /(2 \pi)$ is the value of the Fermi distribution. The results were obtained using a Slater-Jastrow-backflow wave function and a variety of system sizes with $N \geq 50$ in each case. Twist averaging was not used. For comparison, we have plotted the MD obtained by Tanatar and Ceperley (Ref. 3) (open symbols) and Eq. (8.133) of Ref. 10 (dotted lines). 

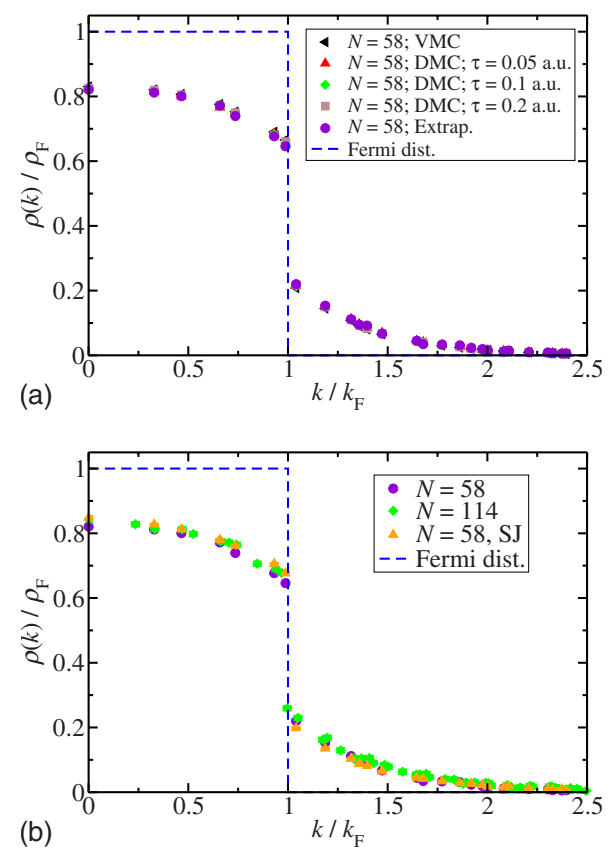

FIG. 4. (Color online) MD $\rho(k)$ for paramagnetic Fermi fluids at $r_{s}=5$ a.u. obtained using VMC and DMC at different system sizes $N$. Backflow was used, except for the data labeled as SJ. The upper panel shows the results obtained at $N=58$ using VMC and DMC with different time steps $\tau$. The lower panel shows the effect of changing the system size and using backflow on the extrapolated MD. Twist averaging was not used. $k_{F}=\sqrt{2} / r_{s}$ is the Fermi wave vector of the paramagnetic fluid and $\rho_{F}=r_{s}^{2} /(2 \pi)$ is the value of the Fermi distribution.

$\rho=\frac{1}{2}\left\{\begin{array}{l}a_{0}+a_{1} x+a_{2} x^{2}+a_{3} x^{3}+a_{4} x^{4} \quad \text { if } x<\sqrt{2} \\ \frac{4 g(0) r_{s}^{2}}{x^{6}}+\left(a_{7}+a_{8} x+a_{9} x^{2}\right) \exp \left[-\frac{(x-\sqrt{2})^{2}}{a_{6}^{2}}\right] \text { if } x>\sqrt{2},\end{array}\right.$

where $x=r_{s} k$ and $a_{i}$ are fitting parameters. $g(0)$ is the contact PCF, which we evaluated using Eq. (9). The fitted parameters

TABLE IV. Fitting parameters in Eq. (8) for the MD and discontinuity $Z$ in the MD at the Fermi edge. The MD is normalized such that the Fermi distribution is 1 .

\begin{tabular}{lcccc}
\hline \hline$r_{s}$ \\
(a.u.) & 1 & 5 & 10 & 30 \\
\hline$a_{0}$ & 1.950 & 1.649 & 1.410 & 0.9745 \\
$a_{1}$ & -0.07342 & -0.03899 & 0.3366 & -0.0535 \\
$a_{2}$ & 0.2805 & 0.07418 & -1.199 & 0.1509 \\
$a_{3}$ & -0.3884 & -0.1920 & 1.148 & -0.3888 \\
$a_{4}$ & 0.1365 & 0.02198 & -0.4363 & 0.1473 \\
$a_{6}$ & 1.171 & 1.017 & 1.035 & 1.379 \\
$a_{7}$ & 0.1648 & 1.682 & 2.091 & 1.428 \\
$a_{8}$ & -0.1135 & -1.282 & -1.566 & -0.8264 \\
$a_{9}$ & 0.02119 & 0.2773 & 0.3438 & 0.1599 \\
$Z$ & 0.866 & 0.398 & 0.209 & 0.0555 \\
\hline \hline
\end{tabular}

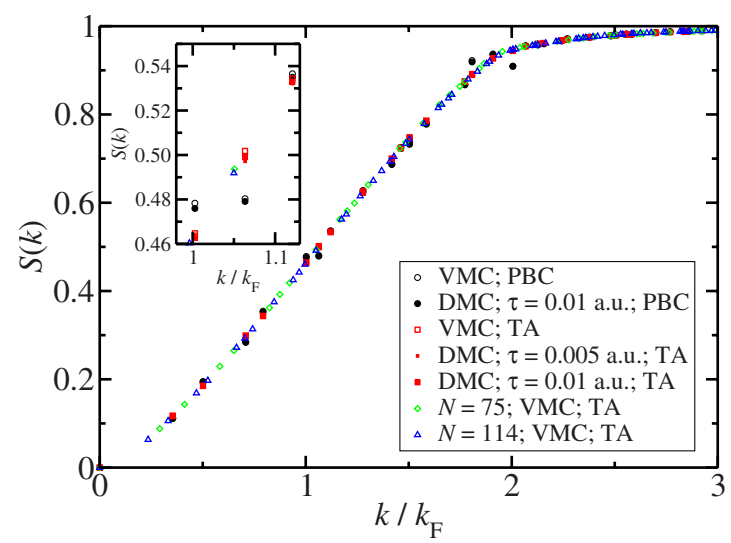

FIG. 5. (Color online) SSF $S(k)$ for a paramagnetic HEG of density parameter $r_{s}=1$ a.u. $k_{F}=\sqrt{2} / r_{s}$ is the Fermi wave vector of the paramagnetic fluid. The system size was $N=50$ electrons, except where indicated otherwise. Results obtained with $\mathbf{k}_{s}=\mathbf{0}$ ("PBC") and "TA" are shown. A Slater-Jastrow-backflow wave function was used in each case. The inset shows in greater detail one of the regions in which the twist-averaged and nontwist-averaged data disagree.

are given in Table IV. Our values for the discontinuity at the Fermi edge are slightly smaller than those reported in Ref. 10.

\section{SSFs}

VMC and DMC SSFs of a 50-electron paramagnetic HEG at $r_{s}=1$ a.u. are shown in Fig. 5. It can be seen that the difference between the VMC and DMC data is in most cases smaller than the difference between the two sets of DMC data, implying that the errors due to extrapolated estimation are small. On the other hand, the difference between the data with $\mathbf{k}_{s}=\mathbf{0}$ and the twist-averaged data is significant. In particular, the former has some unusual features close to integer

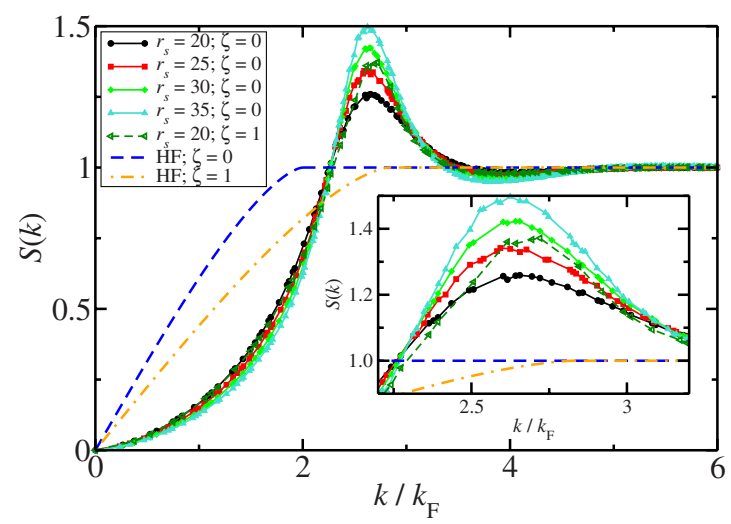

FIG. 6. (Color online) Extrapolated SSF $S(k)$ for HEGs of density parameter $r_{s}$ and spin polarization $\zeta . k_{F}=\sqrt{2} / r_{s}$ is the Fermi wave vector of the paramagnetic fluid. The results were obtained using a Slater-Jastrow-backflow wave function and twist averaging. System sizes of $N=90,114,90$, and 114 were used in the paramagnetic calculations at $r_{s}=20,25,30$, and 35 a.u., respectively; and a system size of $N=45$ was used in the ferromagnetic calculation at $r_{s}=20$ a.u. The inset shows the peak in greater detail. 


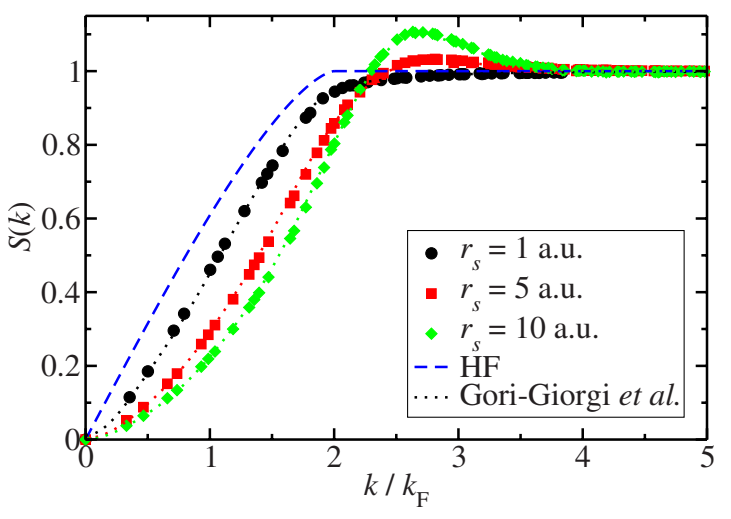

FIG. 7. (Color online) Extrapolated SSF $S(k)$ for paramagnetic HEGs at high density. $k_{F}=\sqrt{2} / r_{s}$ is the Fermi wave vector. SlaterJastrow-backflow wave functions and twist averaging were used. The system sizes are $N=50,58$, and 58 at $r_{s}=1,5$, and 10 a.u., respectively. The curve marked HF shows the Hartree-Fock SSF. The dotted lines show the SSF obtained by taking the Fourier transform of the PCF data of Gori-Giorgi et al. (Ref. 7).

multiples of the Fermi wave vector, one of which is shown in the inset to Fig. 5. Elsewhere, twist averaging has only a small effect. At all densities we find that the twist-averaged $\mathrm{SSF}$ in a 50- or 58-electron cell is in agreement with the SSF in a 114-electron cell, as can be seen in Fig. 5. Since the statistical errors are less significant at the smaller system sizes, we have used $N=50$ or 58 electrons in the high-density data reported below.

The SSFs of paramagnetic and ferromagnetic fluids at low density are shown in Fig. 6, while the SSFs of paramagnetic fluids at high density are shown in Fig. 7. It can be seen that a peak in the SSF at about $k=2.62 k_{F}$ appears at low density, perhaps due to incipient Wigner crystallization. Our SSFs are in good agreement with those of Gori-Giorgi et al. ${ }^{7}$

\section{PCFs}

We compare PCFs obtained at different system sizes using different QMC methods for a paramagnetic HEG at $r_{s}$

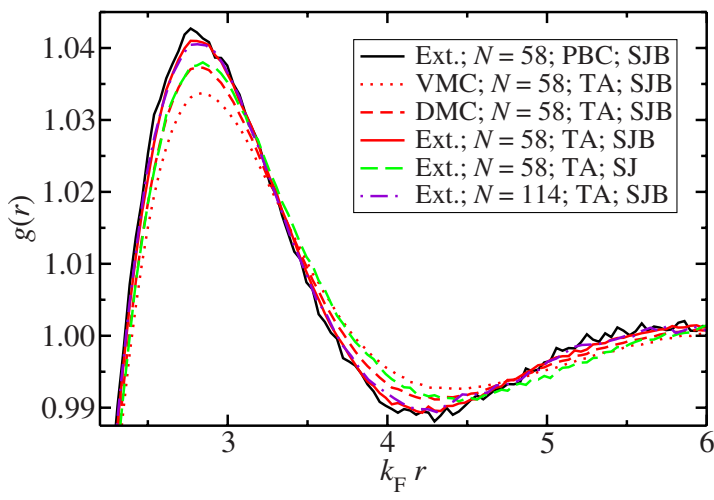

FIG. 8. (Color online) VMC, DMC, and extrapolated ("Ext.") PCFs $g(r)$ for a paramagnetic Fermi fluid of density parameter $r_{s}$ $=5$ a.u. at different system sizes $N$. Twist averaging was used in the curves labeled TA but not in the one labeled PBC. Slater-Jastrow and Slater-Jastrow-backflow wave functions were used in the curves labeled SJ and SJB, respectively. $k_{F}=\sqrt{2} / r_{s}$ is the Fermi wave vector of the paramagnetic fluid.

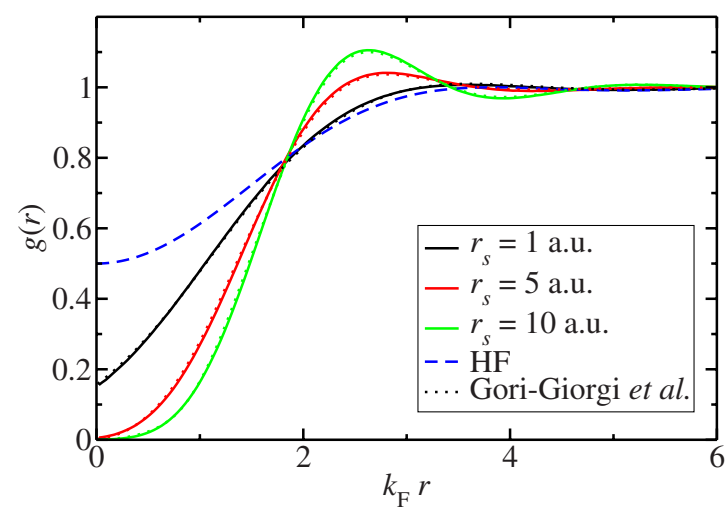

FIG. 9. (Color online) Extrapolated total PCF $g(r)$ for paramagnetic Fermi fluids of density parameter $r_{s} . k_{F}=\sqrt{2} / r_{s}$ is the Fermi wave vector of the paramagnetic fluid. HF stands for Hartree-Fock theory. Twist averaging was used and the QMC calculations were performed at system sizes of $N=50,58$, and 58 electrons at $r_{s}=1,5$, and 10 a.u. The dotted lines show the data of Gori-Giorgi et al. (Ref. 7), which are almost indistinguishable from our data.

$=5$ a.u. in Fig. 8. The difference between the VMC and DMC PCFs, and the difference between extrapolated PCFs obtained with and without backflow are small, implying that the error in the extrapolated PCF is small. Twist averaging has a small effect on the PCF, but the twist-averaged PCFs at $N=58$ and $N=114$ electrons are very similar, implying that the finite-size error in the twist-averaged PCF at $N=58$ is small. We have also verified that the PCF is converged with respect to the DMC time step.

Our PCFs are shown in Fig. 9 (high density) and Fig. 10 (low density), along with the results of Gori-Giorgi et al. ${ }^{7}$ Our PCFs are in good agreement with those of Gori-Giorgi et $a l^{7}$ (as expected, from the SSF results in Fig. 7).

The contact PCF $g(0)$ is especially important in the construction of generalized-gradient-approximation exchangecorrelation functionals. ${ }^{10}$ We give our $g(0)$ values in Table V, and we plot $r_{s} g(0)$ against $r_{s}$ in Fig. 11, along with some other results in the literature. Because our PCFs have converged with respect to system size, and our VMC and DMC results agree with each other when backflow is used, we have simply averaged our VMC and DMC $g(0)$ data at different system sizes in order to reduce the statistical noise in our estimate of the contact PCF. Our results are in reasonably good agreement with the fit to the earlier QMC data of Gori-

TABLE V. Contact PCF of paramagnetic HEGs at five different densities. Twist-averaged VMC and DMC results obtained at different system sizes were averaged to obtain these data.

\begin{tabular}{ll}
\hline $\begin{array}{l}r_{s} \\
\text { (a.u.) }\end{array}$ & \multicolumn{1}{c}{$g(0)$} \\
\hline 1 & $0.1517(4)$ \\
3 & $0.0227(2)$ \\
5 & $0.0050(3)$ \\
7 & $0.00125(5)$ \\
10 & $0.00024(2)$ \\
\hline
\end{tabular}




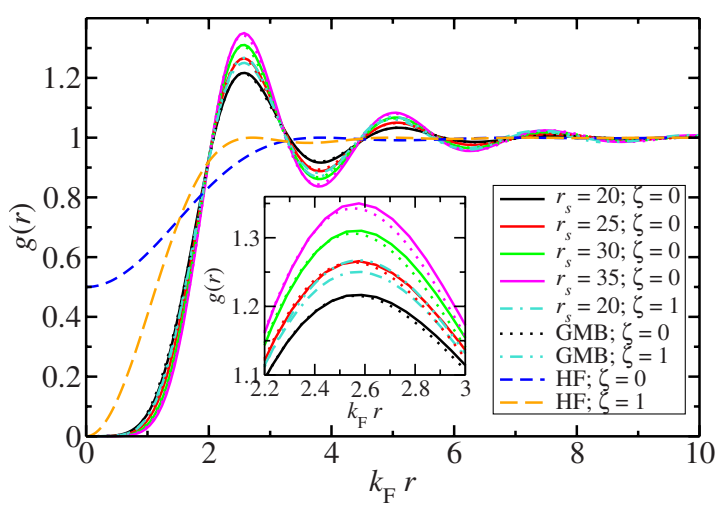

FIG. 10. (Color online) Extrapolated total PCF $g(r)$ for Fermi fluids of density parameter $r_{s}$ and spin polarization $\zeta . k_{F}=\sqrt{2} / r_{s}$ is the Fermi wave vector of the paramagnetic fluid. "GMB" denotes the work of Gori-Giorgi et al. (Ref. 7) (shown by dotted lines), while HF stands for Hartree-Fock theory. Twist averaging was used and the QMC calculations were performed at system sizes of $N$ $=90,114,90$ and 114 at $r_{s}=20,25,30$, and 35, respectively.

Giorgi et al. ${ }^{7}$ and also with the expression for $g(0)$ obtained using ladder theory by Nagano et al. ${ }^{24}$ Interestingly, our results clearly disagree with the more recent calculation of $g(0)$ within ladder theory by Qian, ${ }^{8}$ which involved fewer approximations than the work of Nagano et al. ${ }^{24}$ The close agreement between our results and those of Nagano et al. ${ }^{24}$ must therefore be regarded as a coincidence. The fact that our QMC calculations, using a different trial wave function, are consistent with the data of Gori-Giorgi et al. ${ }^{7}$ strongly suggests that the QMC results for $g(0)$ are reliable, whereas ladder theory is of limited use at low densities. Our results are also in clear disagreement with the formula proposed by Polini et al. ${ }^{25}$ which interpolates between the results of the ladder theory at high density (where it should be exact) and a partial-wave analysis at low density.

The fit to our $g(0)$ data shown in Fig. 11 is

$$
g(0)=\frac{1}{2}\left\{\begin{array}{l}
\left(1+A r_{s}+B r_{s}^{2}\right) \exp \left(-E r_{s}\right) \quad r_{s} \geq 1, \\
1+a r_{s}+b r_{s}^{2}+c r_{s}^{3} \quad r_{s}<1
\end{array}\right.
$$

where $A=-0.25724, B=0.071116$, and $E=0.98553$ were obtained by fitting. Polini et al. ${ }^{25}$ showed that $\lim _{r_{s} \rightarrow 0} g(0)$ $=(1 / 2)\left[1-1.372 r_{s}\right]$, so we have set $a=-1.372$ and deter-

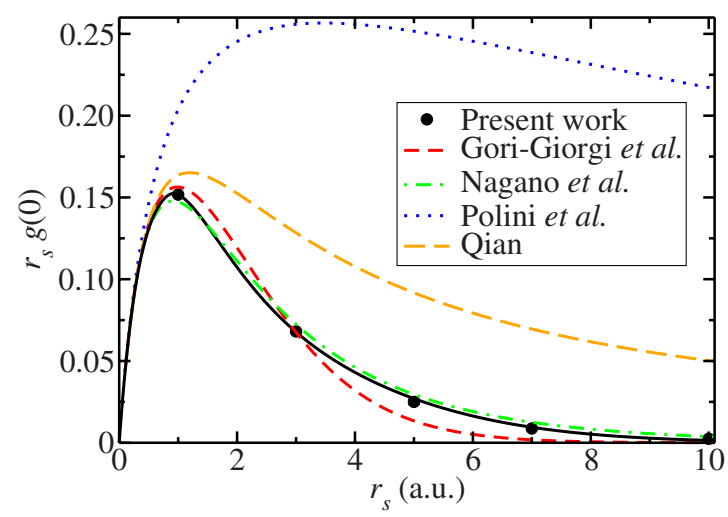

FIG. 11. (Color online) Contact PCF $g(0)$ against density parameter $r_{s}$ as calculated by different authors: the present work (see Table V), Gori-Giorgi et al. (Ref. 7), Nagano et al. (Ref. 24), Polini et al. (Ref. 25), and Qian (Ref. 8).

mined $b=0.997618888$ and $c=-0.3218467056$ by matching the value and derivative of $g(0)$ at $r_{s}=1$ a.u.. ${ }^{26}$

\section{CONCLUSIONS}

We have studied the ground-state properties of the fluid phases of the 2D HEG using QMC. We used highly accurate trial wave functions and dealt with finite-size effects by twist averaging. Twist averaging removes some strange features in the SSF, but our PCFs and SSFs are in good agreement with analytic fits to earlier QMC data, ${ }^{7}$ confirming the accuracy of these formulas. Our MDs show some qualitative differences from earlier QMC results ${ }^{3}$ however; in particular, we do not observe an increase in the MD as the Fermi edge is approached at low density. Finally, we have reported DMC energy data for the high-density 2D HEG, which we used to construct an exchange-correlation functional for 2D DFT calculations.

\section{ACKNOWLEDGMENTS}

We acknowledge financial support from Jesus College, Cambridge, and the UK Engineering and Physical Sciences Research Council (EPSRC-GB). Computing resources were provided by the Cambridge High Performance Computing Service and HPCx.
${ }^{1}$ D. M. Ceperley and B. J. Alder, Phys. Rev. Lett. 45, 566 (1980).

${ }^{2}$ W. M. C. Foulkes, L. Mitas, R. J. Needs, and G. Rajagopal, Rev. Mod. Phys. 73, 33 (2001).

${ }^{3}$ B. Tanatar and D. M. Ceperley, Phys. Rev. B 39, 5005 (1989).

${ }^{4}$ F. Rapisarda and G. Senatore, Aust. J. Phys. 49, 161 (1996).

${ }^{5}$ N. D. Drummond and R. J. Needs (unpublished).

${ }^{6}$ Y. Kwon, D. M. Ceperley, and R. M. Martin, Phys. Rev. B 58, 6800 (1998).

${ }^{7}$ P. Gori-Giorgi, S. Moroni, and G. B. Bachelet, Phys. Rev. B 70, 115102 (2004).
${ }^{8}$ Z. Qian, Phys. Rev. B 73, 035106 (2006).

${ }^{9}$ S. Conti, Ph.D. thesis, Scuola Normale Superiore di Pisa, 1997.

${ }^{10} \mathrm{G}$. F. Giuliani and G. Vignale, Quantum Theory of the Electron Liquid (Cambridge University Press, Cambridge, 2005); Errata: http://www.missouri.edu/ physvign/errata.pdf.

${ }^{11}$ R. J. Needs, M. D. Towler, N. D. Drummond, and P. López Ríos, CASINO version 2.1 User Manual (University of Cambridge, Cambridge, 2008).

${ }^{12}$ J. B. Anderson, J. Chem. Phys. 65, 4121 (1976).

${ }^{13}$ N. D. Drummond, M. D. Towler, and R. J. Needs, Phys. Rev. B 
70, 235119 (2004).

${ }^{14}$ P. López Ríos, A. Ma, N. D. Drummond, M. D. Towler, and R. J. Needs, Phys. Rev. E 74, 066701 (2006).

${ }^{15}$ C. J. Umrigar, K. G. Wilson, and J. W. Wilkins, Phys. Rev. Lett. 60, 1719 (1988).

${ }^{16}$ N. D. Drummond and R. J. Needs, Phys. Rev. B 72, 085124 (2005).

${ }^{17}$ C. J. Umrigar, J. Toulouse, C. Filippi, S. Sorella, and R. G. Hennig, Phys. Rev. Lett. 98, 110201 (2007).

${ }^{18}$ G. Rajagopal, R. J. Needs, S. Kenny, W. M. C. Foulkes, and A. James, Phys. Rev. Lett. 73, 1959 (1994); G. Rajagopal, R. J. Needs, A. James, S. D. Kenny, and W. M. C. Foulkes, Phys. Rev. B 51, 10591 (1995).

${ }^{19}$ Y. Kwon, D. M. Ceperley, and R. M. Martin, Phys. Rev. B 48, 12037 (1993).

${ }^{20}$ C. Lin, F. H. Zong, and D. M. Ceperley, Phys. Rev. E 64,
016702 (2001).

${ }^{21}$ C. Attaccalite, S. Moroni, P. Gori-Giorgi, and G. B. Bachelet, Phys. Rev. Lett. 88, 256601 (2002); 91, 109902(E) (2003).

${ }^{22}$ S. Baroni and S. Moroni, Phys. Rev. Lett. 82, 4745 (1999).

${ }^{23}$ N. D. Drummond, R. J. Needs, A. Sorouri, and W. M. C. Foulkes, Phys. Rev. B 78, 125106 (2008).

${ }^{24}$ S. Nagano, K. S. Singwi, and S. Ohnishi, Phys. Rev. B 29, 1209 (1984); 31, 3166(E) (1985).

${ }^{25}$ M. Polini, G. Sica, B. Davoudi, and M. P. Tosi, J. Phys.: Condens. Matter 13, 3591 (2001).

${ }^{26}$ One can satisfy $g(0)=(1 / 2)\left[1-1.372 r_{s}+O\left(r_{s}^{2}\right)\right]$ by imposing $E$ $=A+1.372$ in Eq. (9); however, we found the corresponding fits to be poor because it is inappropriate to use the high-density expansion to determine the exponent which controls how rapidly $g(0)$ falls off at low density. 